\title{
Case Based Discussion of Surgical Approach to Deep Infiltrating Endometriosis
}

\author{
Hasib Ahmed* \\ Medway NHS Foundation Trust, UK
}

*Corresponding author: Hasib Ahmed, Divisional Director, Women's \& Children's, Medway NHS Foundation Trust, UK.

Received Date: January 05, 2019

Published Date: January 11, 2019

\section{Introduction}

Endometriosis presents a diagnostic challenge as clinical symptoms do not correlate well with the extent of disease [1]. Cramer, et al. [2] found that menstrual cycle length shorter than 27 days, menses longer than 7 days and severe cramping dysmenorrhea were predictive of endometriosis with relative risks of 2.1 (95\% CI 1.5-2.9), 2.4 (95\%CI 1.4-4.0) and 6.7(95\%CI 4.4-10.2) respectively. The study compared 268 women with infertility and laparoscopically confirmed endometriosis with 3794 women admitted for delivery (controls) using a retrospective questionnaire. The study was limited by recall bias and the criteria for laparoscopic diagnosis were not clearly defined. No significant correlation was found with chronic pelvic pain. The issue has been studied prospectively $[3,4]$ in 134 women scheduled for laparoscopy for chronic pelvic pain (CPP). Dyschezia, dyspareunia, and non-menstrual pain were all identified as predictors of deep infiltrating endometriosis (DIE) with odds ratios of 3.9 (95\%CI 1.7-8.9), 4.6 (95\%CI 1.5-14.2) and 2.5 (95\%CI 1.1-5.6) respectively. Mrs. SF presented with all of these symptoms to a greater or lesser extent. One criticism of Chapron's study is that the diagnosis was made on laparoscopic appearance without histological confirmation. Visualization alone has been shown to have a positive predictive value (PPV) for endometriosis of $45 \%$ and up to $36 \%$ of lesions were down staged on histology [5].

\section{Case Report}

Mrs. SF, a 36-year-old nulliparous lady, gave a 20-month history of dysmenorrhea, dyspareunia and dyschezia. Examination revealed a tender nodule high in the pelvis. A diagnosis of rectovaginal endometriosis had been made at diagnostic laparoscopy 18 months previously. She elected to undergo surgery to excise the endometriosis. The risks of injury to the ureters, other organs and vessels were discussed including the small risk of an undiagnosed bowel injury requiring temporary colostomy. Mrs. SF gave her informed consent. She received pre-operative bowel preparation (Citrimag ${ }^{\circledR}$ Sanochemia UK) and on table distal colonic washout (chlorhexidine $0.05 \%$ ). A colorectal surgeon was available. A closed laparoscopy was performed under GA with the patient in modified Lloyd-Davis position. A Spackmann uterine manipulator was used. Two $5 \mathrm{~mm}$ accessory lateral ports were inserted under direct vision. Inspection confirmed endometriosis involving both uterosacral ligaments (USLs). The Pouch of Douglas (PoD) was obliterated with the course of both ureters pulled medially toward an endometriotic nodule. Insertion of a rectal probe with anteversion of the uterus allowed indirect palpation of the nodule in the midline and to the left. Both ovaries and tubes were normal. The course of both ureters was pulled medially toward the endometriotic nodule. Both ovaries and tubes were normal. A normal area of peritoneum on the left ovarian fossa above the ureter was opened with $90 \mathrm{w}$ monopolar diathermy on scissors and medial counter-traction. The peritoneum was cleared from the underlying ureter using cold scissors and blunt dissection. This process was continued until the ureter was released laterally. The affected peritoneum was reflected anteromedially with monopolar and blunt dissection to open the proximal left USL allowing access to the left pararectal space. Peritoneum in the right ovarian fossa was similarly reflected medially releasing the ureter laterally. The rectovaginal nodule was dissected in continuity. The rectovaginal space was entered bilaterally with a mixture of sharp and blunt dissection. A 90w monopolar cutting diathermy spark on scissors was used to enter the rectovaginal septum in the midline. Using a combination of counter traction on the nodule and monopolar dissection of the nodule off the rectum disease was removed with USLs in continuity. The vagina was inadvertently entered and sutured laparoscopically. Hemostasis was confirmed. The raw surface was coated with Sprayshield ${ }^{\mathrm{TM}}$ (Covidien UK). Rectal gas check was negative. Closure was routine. 
Mrs. EM, a 32-year-old nulligravida, presented with 1-week history of worsening lower abdominal pain. Appendicectomy was her only past medical history. She reported a regular menstrual cycle and described cramping period pains. She and her husband were trying for a pregnancy. Urinary $ß$ Hcg was negative. There was lower abdominal tenderness with rebound tenderness in the left iliac fossa. Pelvic examination revealed an exquisitely tender cystic mass to the left of the midline with reduced mobility. The working diagnosis was of an ovarian cyst accident and the patient was prepared for emergency laparoscopy under general anesthetic. Informed consent was obtained for laparoscopy proceeding to ovarian cystectomy/oophorectomy if indicated. The patient was placed in modified Lloyd Davis position, cleaned, draped and catheterized. Open laparoscopy with a disposable Hasson trocar was performed. A 15-mmHg pneumoperitoneum was established and two $5 \mathrm{~mm}$ lateral ports and one $10-12 \mathrm{~mm}$ suprapubic port were placed under direct vision. There was a $7 \mathrm{~cm}$ diameter left sided ovarian endometrioma applied to the left pelvic sidewall and firmly attached to the left uterosacral ligament. The right ovary contained a corpus luteum. The endometrioma was bluntly mobilized. Grasping the left ovarian suspensory ligament caused bleeding which was controlled with bipolar diathermy at $40 \mathrm{w}$. The antimesenteric surface of the endometrioma was opened and the capsule enucleated with a combination of sharp and blunt dissection. The endometrioma was drained of old blood. The cyst capsule was removed in a disposable bag (Endocatch $^{\text {TM }}$ Tyco Hampshire). Closure was routine. An endometriotic cyst was confirmed histologically. Mrs. EM's pain recurred 9 months after surgery. She is currently on an assisted conception program.

\section{Discussion}

On examination a tender nodule was palpable high on the left uterosacral ligament. In a retrospective cohort study logistic regression analysis of signs and symptoms in patients with endometriosis showed tenderness in the Pouch of Douglas (PoD) was associated with a $75 \%$ chance of DIE. (Wright JT personal communication). The clinical features of Mrs. SF were suspicious of DIE and this was confirmed at a previous laparoscopy. One could argue that surgical treatment of endometriosis could have been performed at her first laparoscopy rather than the 2-stage treatment she received. Excisional surgery at first laparoscopy showed improvement in $80 \%$ of the treatment group at 12 months versus 32 in the placebo group in an RCT of 39 women [6]. However, surgery for DIE is technically challenging with particular risks of injury to the large bowel and ureters. On this basis assessment of the extent of disease by laparoscopy is justified to inform discussion with the patient. Furthermore, laparoscopies are negative in 35\% of women with CPP [7]so one step management may not be appropriate. The ureters were dissected laterally away from the nodule as described in M05B. A cold scissor technique is preferable to avoid thermal injury. However, if excessive bleeding is encountered, measures to secure hemostasis, such as bipolar diathermy may cause occult injury with delayed presentation. Ureteric stents and collaboration with urological colleagues are helpful, particularly if endometriosis is found to be involve the ureter directly.
Another issue is whether specific non-invasive tests can improve clinical diagnostic accuracy to facilitate treatment at index laparoscopy. There is insufficient data to support transvaginal ultrasound for the diagnosis of DIE [8] but Magnetic Resonance Imaging (MRI) is emerging as a potential modality for non-invasive diagnosis with a PPV of $92.1 \%$ [9]. MRI is an expensive test, which requires special expertise to interpret the images. In selected patients with a convincing clinical picture it may be appropriate to prepare for major excision at index laparoscopy as long as the patient is fully informed.

Having established the diagnosis of DIE the next issue is planning the method and extent of treatment. Ovarian suppression with gonadotrophin releasing hormone analogues results in symptomatic relief during the course of treatment but the effects are temporary with gradual return of symptoms [10]. Surgical excision is established as an effective treatment for rectovaginal disease [11] and the USLs are one of the commonest locations for DIE [12].

In cases of DIE involving the USLs en-bloc dissection of the USLs is an effective treatment option [13,14]. In a cohort of 107 women the left USL was involved almost twice as often as the right $(64.5 \%$ vs. $35.5 \% \mathrm{P}<0.01)$. This leads us on to a discussion as to whether one or both USLs should be removed. In the case described a greater volume of disease was present in the left USL which may support a unilateral approach. However, several authors have reported microscopic endometriotic disease in visibly normal peritoneum [15-17]. The approach in M05A was to excise the USLs en-block while performing a rectal shave of the nodule off the rectum. Ergonomically the patient was positioned in a modified Lloyd Davis position at hip height of the surgeon. The camera stack was positioned adjacent to the patient's right leg with the screen perpendicular to the gaze of the operator. The monopolar electrosurgical technique using $3 \mathrm{~mm}$ Wolf ${ }^{\mathrm{TM}}$ (Richard Wolf UK Ltd) scissors is a variation of that described by Redwine DB [18]. The cutting current was set at $90 \mathrm{w}$ with the scissors passed through the operating channel of $0^{\circ} 10 \mathrm{~mm}$ operating laparoscope. The tip of the Wolf scissor blade was used 'like a pencil' to direct the monopolar spark to the line of dissection. The tissue just on the nodule side was vaporized with counter traction away from the rectum creating a plane of cleavage. The high-power cutting current creates a spark with which tissue is vaporized with reduced depth of thermal spread compared to contact diathermy. The technique allows bloodless dissection of diseased tissue but requires skill in terms of accuracy of placement and speed of movement of the scissor blade.

There are inherent risks to the rectum and urinary tract with such surgery. A readily available colorectal surgeon is desirable in case of inadvertent injury to the bowel. It is routine practice to formally prepare the bowel to minimize the risk of peritoneal contamination in the event of bowel injury. This established practice has recently been challenged by Bucher P, et al. [19] from their RCT involving 153 patients which showed significantly lower morbidity in patients undergoing left sided colonic surgery with no formal bowel preparation compared to those who had received bowel preparation ( $11 \%$ morbidity vs. $24 \%$, p=0.038). The rectal 
gas test with saline in the pelvis and air inserted into the anal canal with a $50 \mathrm{ml}$ bladder syringe is used. Absence of bubbles is taken as confirmation of rectal integrity. In the event of a small injury to the rectum primary repair without resorting to defunctioning colostomy is possible if the bowel has been appropriately prepared. The routine use of prophylactic antibiotics is established practice. In the case described full thickness disease of the vagina led to entry into the vagina which was sutured laparoscopically. The patient experienced leakage of clear fluid from the vagina in the immediate post-operative period. After exclusion of a ureteric or bladder injury the patient was treated conservatively. Histology of the nodule confirmed fibrosis with foci of endometriosis.

Another important debate is around the extent of surgery to excise rectovaginal endometriosis which varies from rectal shaving, as described in M05A, to discoid and segmental resection. Collaboration with a colorectal surgical colleague is invaluable and a multidisciplinary team approach in treatment centers has been advocated for these complex patients $[20,21]$.

In a histological review of 16 patients undergoing full thickness disc resection prior to segmental resection, disc resection alone was associated with residual disease in 43.8\% (95\% CI 19.8-70.1) [22]. How important this incomplete excision would be clinically is unclear. Anastomotic leak, pelvic abscess and rectovaginal (RV) fistulae following segmental resection are recognized complications. In a retrospective review of 95 patients in a single tertiary center undergoing rectal shave (19\%), disc resection (21\%) and laparoscopic assisted low anterior resection (15\%); there were no anastomotic leaks and a re-operation rate by laparotomy of $8 \%$ [23]. In a retrospective cohort of 128 patients with histologically proven rectovaginal endometriosis and conservative laser surgery in a multidisciplinary setting; 32 women required rectal repair including 3 segmental resections [24]. Three women had RV fistulae requiring laparotomy and defunctioning colostomy including 1 requiring ureteric re-implantation for uretero-vaginal fistula. One patient required ureteric stenting. In the 56 women followed locally $12.5 \%$ reported cure with a further $73.5 \%$ reporting significant improvement. The authors conclude that a conservative approach to surgery for rectovaginal endometriosis gives similar results when compared to radical surgery but with fewer complications. The recommendation for treatments in 'centers of excellence' is reiterated and is the likely way forward.

Mrs. EM was a case of symptomatic endometrioma treated by laparoscopic stripping of the capsule. An anonymous survey of the surgical approach to this problem to 1240 UK specialist gynecologists showed that management is far from uniform [25]. Among 651 respondents (52.5\%) 487 (74.8\%) adopted a surgical approach: $42.3 \%$ by laparotomy with ovarian cystectomy as the usual (94.7\%) procedure. The 249 (56\%) who adopted a laparoscopic approach were divided between fenestration (61\%) and excision (53\%). That almost half the respondents in Jones' survey resorted to laparotomy may be due to lack of training, lack of equipment or both. The same reasons may explain why the $61 \%$ adopting a laparoscopic approach only treated with fenestration. Stripping of the capsule is more challenging and associated with more blood loss particularly around the ovarian hilum. There are a number of theories put forward to explain the origin of endometriomata. Sampson JA [26] first put forward the theory that endometriomata were the sequelae of retrograde menstruation resulting in implantation of endometrial cells on the peritoneum and ovarian cortex. Invagination of the ovarian cortex and occult menstruation from the ectopic endometrium would then give rise to blood filled spaces in the ovary. If this represents the etiology, then fenestration and drainage would be the correct treatment to preserve ovarian cortex. Alternative explanations include invaginated epithelial inclusions arising in coelomic metaplasia [27] and secondary involvement of functional ovarian cysts [28]. If the latter explanations represent the process correctly a cyst capsule should be identifiable which should be amenable to stripping. In practice a cyst capsule is usually identifiable and lends itself to removal as in M05C. If a capsule is not identifiable, or attempted stripping is very difficult and causes excessive haemorrhage, fenestration and bipolar ablation is resorted to in my practice. Normal ovarian stroma containing primordial follicles has been histologically confirmed in $68.9 \%$ of excised capsules of cysts when the cysts stripped [29]. However, the tissue may not be morphologically normal [30]. For women where future fertility is important, such as M05C, loss of follicles may be potentially harmful. Prior ovarian cystectomy is associated with reduced response to stimulated cycles [31,32], and when both ovaries are treated simultaneously; premature ovarian failure postoperatively has been reported in 2.4\% (95\% CI 0.5\%-6.8\%) [33]. This should be borne in mind when counseling patients but should be weighed up against the risk of recurrence of endometriomata which, in one review, appears 3 times as likely after coagulation compared to stripping (OR 3.01: 95\% CI 1.78-5.36; [34]. Vercellini's review included only 1 RCT [35] and 2 of the other studies were retrospective. The issue has been the subject of a more recent Cochrane Systematic Review [36]. The meta-analysis was restricted to 2 RCTs [37] involving a total of 164 patients and demonstrates that excision compared to fenestration and ablation appears to confer a reduction in the recurrence of: dysmenorrhea (OR 0.15 CI 0.06-0.38); dyspareunia (OR 0.14 CI 0.05- 0.44) ; non-menstrual pelvic pain (OR 0.10 CI 0.020.56 ): recurrent endometriomata (OR $0.4195 \% \mathrm{CI} 0.18-0.93$ ) and is associated with an improved 12 month spontaneous pregnancy rate (OR 5.24 CI 1.92-14.27). The studies were not blinded and therefore potentially confounded by assessment and response bias. In addition, the patients in both studies were a mixture of stage III and stage IV suggesting extraovarian disease but whether this was dealt with concomitantly is not clear in the Beretta study. Extraovarian disease was treated in the Alborzi study so differences in postoperative pain scores may not necessarily have been due to differences in the surgical approach to endometriomata. Indeed, extraovarian disease was present in Mrs. EM which is a common association, but this was not radically excised. This could be considered as incomplete treatment, but the aim of the treatment was to alleviate the patient's acute symptoms, which was pain and tenderness. Future pregnancy was desired, and treatment was tailored to her individual needs. In addition, the patient had not been counselled regarding treatment to other disease in the pelvis. On reflection whenever an endometrioma is suspected preoperative discussion should include the possibility of treatment of disease 
elsewhere. This is my preferred practice. However, patients are not given mechanical bowel preparation routinely unless there are convincing symptoms or signs of rectovaginal disease (dyschezia, cyclical rectal bleeding or the presence of palpable tender disease in the USLs or RV septum).

\section{Acknowledgement}

None.

\section{Conflict of Interest}

No conflict of interest.

\section{References}

1. Porpora MG, Koninckx PR, Piazze J, Natili M, Colagrande S, et al. (1999) Correlation between endometriosis and pelvic pain. J Am Assoc Gynecol Laparosc 6(4): 429-434.

2. Cramer DW, Wilson E, Stillman RJ, Berger MJ, Belisle S, et al. (1986) The relation of endometriosis to menstrual characteristics, smoking and exercise. J Am Med Assoc 255(14): 1904-1908.

3. Chapron C, Barakat H, Fritel X, Dubuisson JB, Bréart G, et al. (2004) Presurgical diagnosis of posterior deep endometriosis based on a standardised quesstionnaire. Hum Reprod 20(2): 507-513.

4. Chapro C, Fauconnier A, Dubuisson JB, Vieira M, Bonte H, et al. (1997) Does deep endometriosis infiltrating the uterosacral ligaments present an asymmetric lateral distribution? BJOG 108(10): 1021-1024.

5. Redwine DB, Wright JT (2001) Laparoscopic treatment of complete obliteration of the cul-de-sac associated with endometriosis: long-term follow-up of en bloc resection. Fertil Steril 76(2): 358-365.

6. Abbot J, Hawe J, Hunter D, Holmes M, Finn P, et al. (2004) Laparoscopic excision of endometriosis: a randomized, placebo-controlled trial. Fertil Steril 82(4): 878-884.

7. Howard F (2000) Endoscopy in pelvic pain, diagnosis and management Lippincott Williams and Wilkins, Philadelphia, USA, p. 63.

8. Bazot M, Thomassin I, Hourani R, Cortez A, Darai E (2004) Diagnostic accuracy of transvaginal sonography for deep pelvic endometriosis. Ultrasound Obstet Gynecol 24(2): 180-185.

9. Bazot M, Darai E, Hourani R, Thomassin I, Cortez A, et al. (2004) Deep pelvic endometriosis: MR imaging for diagnosis and prediction of extension of disease. Radiology 232(2): 379-389.

10. Waller KG, Shaw RW (1993) Gonadotropin-releasing hormone analogues for the treatment of endometriosis: long-term follow-up. Fertil Steril 59(3): 511-515.

11. Donnez J, Nisolle M, Gillerot S, Smets M, Bassil S, et al. (1997) Rectovaginal septum adenomyotic nodules: A series of 500 cases. Br J Obstet Gynaecol 104(9): 1014-1018.

12. Cornillie FJ, Oosterlynck D, Lauweryns JM, Koninckx PR (1990) Deeply infiltrating pelvic endometriosis: histology and clinical significance. Fertil Steril 53(6): 978-983.

13. Chapron C, Dubuisson JB, Fritel X, Fernandez B, Poncelet C, et al. (1999) Operative management of deep endometriosis infiltrating the uterosacral ligaments. J Am Assoc Gynecol Laparos 6(1): 31-37.

14. Garry R, Clayton R, Hawe J (2000) The effect of endometriosis and its radical laparoscopic excision on quality of life indicators. BJOG 107(1): 44-54.

15. Nezhat F, Allan CJ, Nezhat C, Martin DC (1991) Nonvisualized endometriosis at laparoscopy. Int J Fert 36(6): 340-343.

16. Nisolle M, Paindaveine B, Bourdon A, Berlière M, Casanas-Roux F, et al. (1990) Histologic study of peritoneal endometriosis in infertile women. Fertil Steril 53(6): 984-988.

17. Redwine DB, Yocom LB (1990) A serial section study of visually normal pelvic peritoneum in patients with endometriosis. Fertil Steril 54(4): 648-651.
18. Redwine DB (1993) Laparoscopic excision of endometriosis with $3 \mathrm{~mm}$ scissors: Comparison of operating times between sharp excision and electro-excision. J Am Assoc Gynecol Laparosc 1(1): 24-30.

19. Bucher P, Gervaz P, Soravia C, Mermillod B, Erne M, et al. (2005) Randomized clinical trial of mechanical bowel preparation versus no preparation before elective left-sided colorectal surgery. Br J Surg 92(4): 409-414.

20. Kennedy S, Bergqvist A, Chapron C, D’Hooghe T, Dunselman G, etal. (2005) ESHRE guideline for the diagnosis and treatment of endometriosis. Hum Reprod 20(10): 2698-2704.

21. Redwine DB, Wright JT (2001) Laparoscopic treatment of complete obliteration of the cul-de-sac associated with endometriosis: long-term follow-up of en bloc resection. Fertil Steril 76(2): 358-365.

22. Remorgida V, Ragni N, Ferrero S, Anserini P, Torelli P, et al. (2005) How complete is full thickness disc resection of bowel endometriotic lesions? A prospective surgical and histological study. Hum Reprod 20(8): 23172320.

23. Jatan AK, Solomon MJ, Young J, Cooper M, Pathma-Nathan N (2006) Laparoscopic management of rectal endometriosis. Dis Colon Rectum 49(2): 169-174.

24. Slack A, Child T, Lindsey I, Kennedy S, Cunningham C, et al. (2007) Urological and colorectal complications following surgery for rectovaginal endometriosis. BJOG 114(10): 1278-1282.

25. Jones KD, Fan A, Sutton CJ (2002) The ovarian endometrioma: why is it so poorly managed? Indicators from an anonymous survey. Hum Reprod 17(4): 845-849.

26. Sampson JA (1921) Perforating hemorrhagic (chocolate) cysts of the ovary. Arch Surg 3(2): 245-323.

27. Donnez J, Nisolle M, Gillet N, Smets M, Bassil S, et al. (1996) Large ovarian endometriomas. Hum Reprod 11(3): 641-646.

28. Nezhat F, Nezhat C, Allan CJ, Metzger DA, Sears DL (1992) A clinical histological classification of endometriomas: implications for a mechanism of pathogenesis. J Reprod Med 37(9): 771-776.

29. Hachisuga, T, Kawarabayashi, T (2002) Histopathological analysis of laparoscopically treated ovarian endometriotic cysts with special reference to loss of follicles. Hum Reprod 17(2): 432-435.

30. Muzii L, Bianchi A, Crocè C, Manci N, Panici PB (2002) Laparoscopic excision of ovarian cysts: is the stripping technique a tissue-sparing procedure? Fertil.Steril 77(3): 609-614

31. Loh FH, Tan AT, Kumar J, Ng SC (1999) Ovarian response after laparoscopic ovarian cystectomy for endometriotic cysts in 132 monitored cycles. Fertil Steril 72(2): 316-321.

32. Nargund G, Cheng WC, Parsons J (1996) The impact of ovarian cystectomy on ovarian response to stimulation during in-vitro fertilization cycles. Hum Reprod 11(1): 81-83.

33. Busacca M, Riparini J, Somigliana E, Oggioni G, Izzo S, et al. (2006) Postsurgical ovarian failure after laparoscopic excision of bilateral endometriomas. Am J Obstet Gynecol 195 (2): 421-425.

34. Vercellini P, Chapron C, De Giorgi O, Consonni D, Frontino G, et al. (2003) Coagulation or excision of ovarian endometriomas? Am J Obstet Gynecol 188(3): 606-610.

35. Beretta P, Franchi M, Ghezzi F, Busacca M, Zupi E, et al. (1998) Randomized clinical trial of two laparoscopic treatments of endometriomas: cystectomy versus drainage and coagulation. Fertil Steril 70(6): 11761180 .

36. Hart R, Hickey M, Maouris P, Buckett W, Garry R (2005) Excisional surgery versus ablative surgery for ovarian endometriomata: a Cochrane Review. Hum Reprod 20(11): 3000-3007.

37. Alborzi S, Momtahan M, Parsanezhad ME, Dehbashi S, Zolghadri J, et al. (2004) A prospective, randomized study comparing laparoscopic ovarian cystectomy versus fenestration and coagulation in patients with endometriomas. Fertil Steril 82(6): 1633-1637. 